Journal of Universal Mathematics

Vol.5 No.1 PP.24-35 (2022)

ISSN-2618-5660

DOI: $10.33773 /$ jum. 985160

\title{
PERFECT CODES OVER HURWITZ INTEGERS INDUCED BY CIRCULANT GRAPHS
}

\author{
M. GÜZELTEPE, AND G. GÜNER
}

0000-0002-2089-5660 and 0000-0001-7634-3075

\begin{abstract}
In this paper, a new family of $t$-error correcting perfect codes over Hurwitz integers is presented. To obtain these perfect codes, the perfect $t$-dominating sets over the circulant graphs are used. The codewords of such perfect codes are generated by the elements of a subgroup of the considered group.
\end{abstract}

\section{INTRODUCTION}

If a code satisfy the sphere-packing bound in any given metric, then the code called perfect code. Perfect codes are important since perfect codes plays an importance role in coding theory. The first perfect codes which were subspaces of $\mathbb{Z}_{2}^{n}$ were defined by Hamming in [4]. The first non-linear perfect 1-error correcting binary code was constructed by Vasil'ev in [15]. Vasil'ev's construction was generalized to $q$-ary case by Lindström and independently Schönheim in [10, 14]. Group and non-group perfect codes which were not equal to any linear code were given by Heden in [5]. Besides, perfect codes have been investigated with respect to some other metrics such as the Lee metric, the Mannheim metric, the Lipschitz metric. Some perfect codes with respect to the Lee metric introduced in [9]. Huber defined Mannheim metric, and presented perfect 1-Mannheim error correcting codes (shortly OMEC) in the metric in [8]. The dimension of OMEC codes not only $n-1$, but also $n-k(k>1)$ were constructed by Güzeltepe and Heden in [3]. The Lipschitz metric was presented and some perfect codes over Lipschitz integers were introduced with respect to the Lipschitz metric in $[11,12]$. A generalization of perfect Lee-error-correcting codes and perfect 1-error correcting Lipschitz weight codes were presented by Heden and Güzeltepe in $[6,7]$.

The Hurwitz metric was introduced in [1,3]. Besides, Güzeltepe constructed linear codes over Hurwitz integers with respect to the Hurwitz metric for a Hurwitz prime in [1]. These linear codes were not perfect.

Date: Received: 2021-08-20; Accepted: 2022-03-01.

2000 Mathematics Subject Classification. 94B05, 94B60.

Key words and phrases. Circulant graphs, Hurwitz integers, Perfect codes, Quadrature amplitude modulation (QAM) constellations. 
On the other hand, the common trait of the papers $[1,2,3,6]$ is that the perfect codes were obtained by using a chosen prime over relevant structures. Unlike these articles, we use only odd Hurwitz integers being product of distinct primes to construct perfect codes over Hurwitz integers. The main idea in the presented paper is inspired by the article [13]. In that paper [13], a method for defining new metrics over two-dimension signal spaces and families of perfect codes of length one over lattice constellations obtained by Gaussian integers and Eisenstein Jacobi integers were presented by Martínez et al. They mainly considered QAM-like signal spaces and defined a new distance over QAM-like constellations imported from degree-four circulant graphs whose nodes were labeled with Gaussian integers. By means of these graphs, they constructed perfect $t$-correcting codes over Gaussian integers with length one.

The rest of the paper is organized as follows. In Section 2, basic definitions and theorems in Hurwitz integers are given. A connection between Circulant graph and Hurwitz integers is obtained in Section 3. Perfect $t$-dominating sets is defined in Section 4. Using these sets, perfect codes over Hurwitz integers are constructed in that section. In terms of average energy and bandwidth occupancy, a simple comparison between these perfect codes and some perfect codes given in literature is presented in the last section.

\section{On HuRwitz INTEGERS}

In this section, we give some basic definitions and theorems which we need throughout our study.

Definition 2.1 (see [3]). Hamilton Quaternions $\mathcal{H}(\mathbb{R})$ is the free $\mathbb{R}$-module over the symbols $1, i, j, k$ and the set of Hamilton Quaternions is defined as following:

$$
\mathcal{H}(\mathbb{R})=\left\{a_{0}+a_{1} i+a_{2} j+a_{3} k: a_{0}, a_{1}, a_{2}, a_{3} \in \mathbb{R}\right\} .
$$

Here, 1 is the multiplicative identity. Morever,

(1) $i^{2}=j^{2}=k^{2}=-1$ and

(2) $i j=-j i=k ; j k=-k j=i ; k i=-i k=j$.

(3) If $q=a_{0}+a_{1} i+a_{2} j+a_{3} k$ is a quaternion, then its conjugate is denoted by $q^{*}$ and $q *=a_{0}-\left(a_{1} i+a_{2} j+a_{3} k\right)$.

(4) The norm $N(q)$ of $q \in \mathcal{H}(\mathbb{R})$ is $N(q)=q q^{*}=a_{0}^{2}+a_{1}^{2}+a_{2}^{2}+a_{3}^{2}$ and $N\left(q_{1} q_{2}\right)=N\left(q_{1}\right) N\left(q_{2}\right)$, that is, the norm $N$ is a multiplicative norm.

Definition 2.2 (see [3]). The Lipschitz integers $\mathcal{H}(\mathbb{Z})$ is a subset of $\mathcal{H}(\mathbb{R})$ and is defined as

$$
\mathcal{H}(\mathbb{Z})=\left\{a_{0}+a_{1} i+a_{2} j+a_{3} k: a_{0}, a_{1}, a_{2}, a_{3} \in \mathbb{Z}\right\} .
$$

Definition 2.3 (see [2]). The set of Hurwitz integers is the set $\mathcal{H}=\mathcal{H}(\mathbb{Z}) \cup$ $\mathcal{H}\left(\mathbb{Z}+\frac{1}{2}\right)$, that is,

$$
\begin{aligned}
\mathcal{H} & =\left\{a_{0}+a_{1} i+a_{2} j+a_{3} k: a_{0}, a_{1}, a_{2}, a_{3} \in \mathbb{Z} \vee a_{0}, a_{1}, a_{2}, a_{3} \in \mathbb{Z}+\frac{1}{2}\right\} \\
& =\left\{\frac{a_{0}+a_{1} i+a_{2} j+a_{3} k}{2}: a_{0}, a_{1}, a_{2}, a_{3} \in \mathbb{Z}, a_{0} \equiv a_{1} \equiv a_{2} \equiv a_{3}(\bmod 2)\right\} .
\end{aligned}
$$

Definition 2.4 (see [3]). If the norm of a Hurwitz integer $q$ is an odd integer, then the element $q$ is called an odd Hurwitz integer. Similarly, if the norm of a Hurwitz integer $\alpha$ is a prime integer, then the element $\alpha$ is called prime Hurwitz integer. 
In this study, we use only odd Hurwitz integers to construct perfect codes over Hurwitz integers.

Definition 2.5. Let $q_{1}, q_{2}$ be two elements of Hurwitz integers $\mathcal{H}$ and let $\alpha$ be an odd Hurwitz integer. If there exists $\beta \in \mathcal{H}$ such that $q_{1}-q_{2}=\alpha \beta$, then $q_{1}, q_{2} \in \mathcal{H}$ are left congruent modulo $\alpha$ and it is denoted as $q_{1} \equiv_{l} q_{2}(\bmod \alpha)$.

Let $\mathcal{H}_{\alpha}$ denotes the complete set of left coset representatives. In this situation, the elements of $\mathcal{H}_{\alpha}$ are not left congruent each other modulo $\alpha$. Right congruent can be defined like left congruent.

Theorem 2.6 (see [2]). If $\alpha$ is an odd Hurwitz prime, then the size of $\mathcal{H}_{\alpha}$ is equal to $N(\alpha)^{2}$.

Corollary 2.7. Let $0 \neq \alpha$ and $\beta$ be in $\mathcal{H}$ and let $\beta$ be a left-divisor of $\alpha$. Then the subgroup generated by the element $\beta$ is denoted by $\langle\beta\rangle$ and the number of the elements of the subgroup $\langle\beta\rangle$ is equal to $N(\alpha)^{2} / N(\beta)^{2}$.

Proof. $\mathcal{H}_{\alpha}$ is an additive group and $\langle\beta\rangle$ is a subgroup of $\mathcal{H}_{\alpha}$. So, the proof is clear from the Lagrange Theorem.

\section{Circulant graph and Hurwitz integers}

In this section, a connection between circulant graph $C_{N}\left(j_{1}, \ldots, j_{m}\right)$ and $\mathcal{H}_{\alpha}$ is given.

Definition 3.1. The distance $\beta, \gamma \in \mathcal{H}_{\alpha}$ is defined as

$$
d_{\alpha}(\beta, \gamma)=N(\delta)
$$

where $\delta=a_{0}+a_{1} i+a_{2} j+a_{3} k$ denotes an element in the coset $\beta-\gamma$ in $\mathcal{H}_{\alpha}$ with $\left|a_{0}\right|+\left|a_{1}\right|+\left|a_{2}\right|+\left|a_{3}\right|$ minimum. We also define the weight of $\beta \in \mathcal{H}_{\alpha}$ as

$$
w_{\alpha}=d_{\alpha}(\beta, 0) \text {. }
$$

There are 24 elements of weight one in Hurwitz integers $\mathcal{H}$. These elements are $\pm 1, \pm i, \pm j, \pm k$ and $\pm \frac{1}{2} \pm \frac{i}{2} \pm \frac{j}{2} \pm \frac{k}{2}$. From now on $\varepsilon$ denotes the following set:

$$
\left\{ \pm 1, \pm i, \pm j, \pm k, \pm \frac{1}{2} \pm \frac{i}{2} \pm \frac{j}{2} \pm \frac{k}{2}\right\}
$$

By adding the elements of the set $\varepsilon$ one by one to $\gamma \in \mathcal{H}_{\alpha}$, we determine the elements at distance one from exactly $\gamma$.

Definition 3.2. Let $0 \neq \alpha \in \mathcal{H}$ be an odd Hurwitz integer. If we take

(1) $V=\mathcal{H}_{\alpha}$ is the set of vertices (nodes) and

(2) $E=\left\{(\beta, \gamma) \in V \times V: d_{\alpha}(\beta, \gamma)=1\right\}$ is the set of edges, then $G_{\alpha}(V, E)$ defines a graph generated by $\alpha$.

Definition 3.3. (see [13]) A circulant graph with $N$ vertices and jumps $\left\{j_{1}, j_{2}, \cdots, j_{m}\right\}$, where $m<N / 2$, is an undirected graph in which each vertex $n, 0 \leq n \leq N-1$, is adjacent to all the vertices $n \pm j_{i}$, with $1 \leq i \leq m$. We denote this graph as $C_{N}\left(j_{1}, j_{2}, \cdots, j_{m}\right)$.

Theorem 3.4. Let $e_{1} \in\{i, j, k\}$ and let $\alpha=a_{0}+a_{1} i+a_{2} j+a_{3} k=a_{0}+a_{1} i+$ $\left(a_{2}+a_{3} i\right) e_{1} \in \mathcal{H}$ be an odd Hurwitz integer. Then $C_{N(\alpha)^{2}}\left(j_{1}, \ldots, j_{12}\right)$ and $G_{\alpha}$ are isomorphic graphs. 


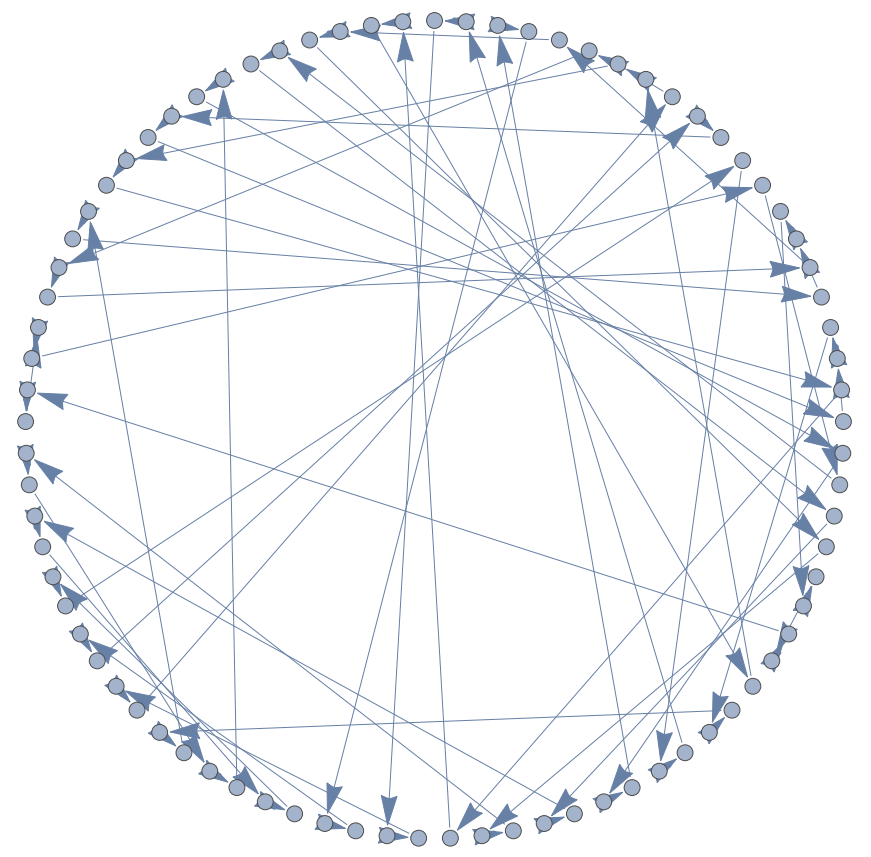

Figure 1. The graph $G_{-1+2 i+2 j}$

$\begin{aligned} \operatorname{In}[4]:= & \text { A }=\text { CirculantGraph@ } 1,813,14,15,16,17,18,19,20,21,22,23,24<D \\ & \text { EdgeCount @ } \mathrm{DAD}\end{aligned}$ GraphDiameter@D

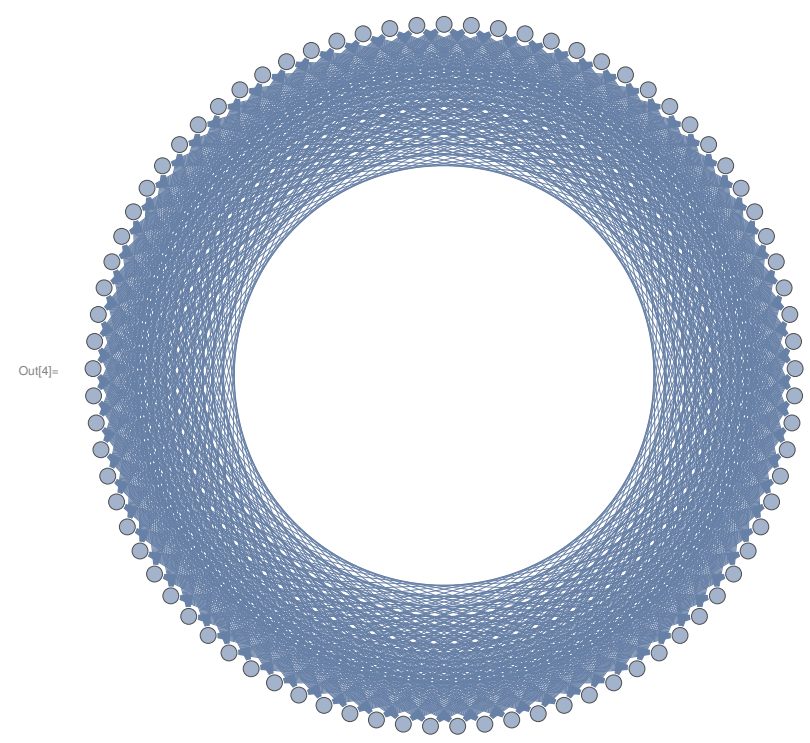

Ou[[5] $=972$

Out[6]=3

Figure 2. The graph $C_{81}(13, \ldots, 24)$ 
Proof. The edges of $G_{\alpha}$ and the edges of $C_{N(\alpha)^{2}}\left(j_{1}, \ldots, j_{12}\right)$ are chosen as the elements of $\mathcal{H}_{\alpha}$ and the elements of $\mathbb{Z}_{N(\alpha)} \times \mathbb{Z}_{N(\alpha)}$, respectively. Therefore, it is sufficient to prove that $\mathcal{H}_{\alpha}$ and $\mathbb{Z}_{N(\alpha)} \times \mathbb{Z}_{N(\alpha)}$ are isomorphic groups. We now consider the function

$$
\begin{gathered}
\psi: \mathbb{Z}_{N(\alpha)} \times \mathbb{Z}_{N(\alpha)} \rightarrow \mathcal{H}_{\alpha} \\
\left(q_{1}, q_{2}\right) \mapsto\left(x_{1}+y_{1}\right) i+\left(x_{2}+y_{2}\right) e_{1}(\bmod \alpha),
\end{gathered}
$$

where $x_{1}, y_{1}, x_{2}, y_{2} \in \mathbb{Z}_{N(\alpha)}, q_{1}=a_{0} x_{1}+a_{1} y_{1}, q_{2}=a_{2} x_{2}+a_{3} y_{2}(\bmod N(\alpha))$. The function $\psi$ is a bijective function. The bases of these groups $\mathcal{H}_{\alpha}$ and $\mathbb{Z}_{N(\alpha)} \times \mathbb{Z}_{N(\alpha)}$ are $e_{2}, e_{3} \in\{1, i, j, k\}$ and $\{(1,0),(0,1)\}$, respectively. Hence we get $\psi((1,0))=$ $e_{2}, \psi((0,1))=e_{3}$, where $\psi((x, y))=\beta_{1} e_{2}+\beta_{2} e_{3}(\bmod \alpha), x, y \in \mathbb{Z}_{N(\alpha)}$ and $\beta_{1}, \beta_{2} \in \mathcal{H}_{\alpha}$. Hence, the proof is completed.

Example 3.5. Let $\alpha=-1+2 i+2 j$. Fig. 1 shows the graph $\mathcal{H}_{\alpha}$ and Fig. 2 shows the graph $C_{81}(13, \ldots, 24)$. The vertexes given in Fig. 1 shows one twelfth of all vertexes. The diameter of these graphs is 3 . This shows that the distance between 0 and the elements of $\mathcal{H}_{\alpha}$ is less than or equal to 3 , that is, $N(q) \leq 3$ for all $q \in \mathcal{H}_{\alpha}$.

\section{Perfect $t$-Dominating Sets and Perfect Codes Over the Hurwitz INTEGERS}

In this paper, we study on arbitrary parameter $t$, give conditions for the existence of perfect $t$-dominating sets.

Proposition 1. If $\alpha$ is a Hurwitz integer and $\rho_{1}, \rho_{2} \in \varepsilon$ then the norm $N(\alpha)$ is equal to the norm $N\left(\rho_{1} \alpha \rho_{2}\right)$.

Proof. Recall that the norm $N$ is a multiplicative norm and $N\left(\rho_{1}\right)=N\left(\rho_{2}\right)=1$ since $\rho_{1}, \rho_{2} \in \varepsilon$. Hence, we have

$$
N\left(\rho_{1} \alpha \rho_{2}\right)=N\left(\rho_{1}\right) N(\alpha) N\left(\rho_{2}\right)=N(\alpha) .
$$

It is clear that if $\alpha_{1}, \cdots, \alpha_{r}$ are odd Hurwitz integers then $\alpha_{1} \ldots \alpha_{r}$ is an odd Hurwitz integer.

Proposition 2. Let $\alpha$ be an odd Hurwitz integer and let $\beta_{1}, \beta_{2} \in \mathcal{H}$. If

$$
\beta_{1}=\beta_{2}(\bmod \alpha),
$$

then

$$
\rho_{1} \beta_{1} \rho_{2}=\rho_{1} \beta_{2} \rho_{2}\left(\bmod \rho_{1} \alpha \rho_{2}\right) .
$$

Proof. If $\beta_{1}=\beta_{2}(\bmod \alpha)$, then we get

$$
\beta_{2}=\beta_{1}+\alpha \delta, \delta \in \mathcal{H} .
$$

Multiplying left sides of the above equation by $\rho_{1}$ and right sides by $\rho_{2}$, we obtain

$$
\begin{array}{rcc}
\rho_{1} \beta_{2} \rho_{2} & = & \rho_{1}\left(\beta_{1}+\alpha \delta\right) \rho_{2}=\rho_{1} \beta_{1} \rho_{2}+\rho_{1}(\alpha \delta) \rho_{2} \\
& = & \rho_{1} \beta_{1} \rho_{2}+\rho_{1}\left(\alpha\left(\rho_{2} \rho_{2}^{-1}\right) \delta\right) \rho_{2} \\
& = & \rho_{1} \beta_{1} \rho_{2}+\rho_{1}\left(\alpha \rho_{2}\right)\left(\rho_{2}^{-1} \delta \rho_{2}\right)=\rho_{1} \beta_{1} \rho_{2}+\left(\rho_{1} \alpha \rho_{2}\right) \delta_{1} .
\end{array}
$$

This shows that

$$
\rho_{1} \beta_{1} \rho_{2}=\rho_{1} \beta_{2} \rho_{2}\left(\bmod \rho_{1} \alpha \rho_{2}\right) .
$$


The proof of next proposition is straightforward from the proof of Prop. 2 .

Proposition 3. Let $\alpha$ be an odd Hurwitz integer. If the set $\left\{\varepsilon_{1}, \varepsilon_{2}, \cdots, \varepsilon_{n}\right\}$ is a partition of $\mathcal{H}_{\alpha}$ then the set $\left\{\rho_{1} \varepsilon_{1} \rho_{2}, \rho_{1} \varepsilon_{2} \rho_{2}, \cdots, \rho_{1} \varepsilon_{n} \rho_{2}\right\}$ becomes a partition of $\mathcal{H}_{\rho_{1} \alpha \rho_{2}}$.

The proof of next lemma is straightforward from Prop. 3 .

Lemma 4.1. $G_{\alpha_{1}} \cong G_{\alpha_{2}}$ if there exist $\rho_{1}, \rho_{2} \in \varepsilon$ such that $\alpha_{1}=\rho_{1} \alpha_{2} \rho_{2}$, where $\alpha_{1}, \alpha_{2} \in \mathcal{H}$.

Definition 4.2. Let $\alpha$ be a Hurwitz integer. A sphere (ball) centered at $\gamma$ with radius $t$ in $G_{\alpha}$ is defined as

$$
B_{t}(\gamma)=\left\{\beta \in \mathcal{H}_{\alpha}: d_{\alpha}(\beta, \gamma) \leq t\right\},
$$

where $t \in \mathbb{N}$. If $q \in \mathcal{H}_{\alpha}$ is in the $B_{t}(\gamma)$, then it is said that the vertex $q$ is $t$-dominated by the vertex $\gamma$.

We give the following definition as in [13].

Definition 4.3. Let a vertex subset $S \subset G_{\alpha}$ and $t \in \mathbb{Z}^{+}$. If every vertex of $G_{\alpha}$ is $t$-dominated by a unique vertex in $S$, then $S$ is called a perfect $t$-dominating set.

Example 4.4. For $\alpha=1+3 i+2 j+k$ and $\gamma=-2 j-k$, the set of $B_{1}(-2 j-k)=$ $\left\{\beta \in \mathcal{H}_{1+3 i+2 j+k}: d_{1+3 i+2 j+k}(\beta,-2 j-k) \leq 1\right\}$ is a sphere centered at $-2 j-k$ with radius 1 in $\mathcal{H}_{1+3 i+2 j+k}$.

If $t=0$, then $d_{1+3 i+2 j+k}(\beta,-2 j-k)=0$. Hence, we get $\beta=-2 j-k$ and $-2 j-$ $k \in B_{1}(-2 j-k)$.

If $t=1$, then $d_{1+3 i+2 j+k}(\beta,-2 j-k)=1$. So, we add all of the elemets of weight one to $\gamma=-2 j-k$ in an effort to determine $1-$ dominating set of $\gamma=-2 j-k$.

For $-1: \beta=(-2 j-k)-1=-1-2 j-k \equiv \frac{1}{2}-\frac{i}{2}+\frac{j}{2}+\frac{3 k}{2}(\bmod 1+3 i+2 j+k)$. Then we get $\frac{1}{2}-\frac{i}{2}+\frac{j}{2}+\frac{3 k}{2} \in B_{1}(-2 j-k)$.

For 1: $\beta=(-2 j-k)+1=1-2 j-k \equiv-\frac{1}{2}+\frac{i}{2}+\frac{3 j}{2}-\frac{k}{2}(\bmod 1+3 i+2 j+k)$. Then we get $-\frac{1}{2}+\frac{i}{2}+\frac{3 j}{2}-\frac{k}{2} \in B_{1}(-2 j-k)$.

By processing similar technique for 24 elements of weight one, we obtain 1-dominating set of $\gamma=-2 j-k \in \mathcal{H}_{\alpha}$ as

$$
\begin{aligned}
& B_{1}(-2 j-k)=\left\{-2 j-k,-\frac{1}{2}+\frac{i}{2}+\frac{3 j}{2}-\frac{k}{2}, \frac{1}{2}-\frac{i}{2}+\frac{j}{2}+\frac{3 k}{2}, \frac{3}{2}+\frac{i}{2}+\frac{j}{2}+\frac{3 k}{2}, 1+2 i,\right. \\
& -j-k,-\frac{3}{2}+\frac{i}{2}+\frac{j}{2}-\frac{k}{2},-2 j, \frac{3}{2}-\frac{i}{2}+\frac{j}{2}+\frac{k}{2}, \frac{1}{2}+\frac{i}{2}-\frac{3 j}{2}-\frac{k}{2}, \\
& -\frac{1}{2}+\frac{i}{2}-\frac{3 j}{2}-\frac{k}{2}, \frac{1}{2}-\frac{i}{2}-\frac{3 j}{2}-\frac{k}{2},-1+i+j, \frac{1}{2}+\frac{i}{2}-\frac{3 j}{2}-\frac{3 k}{2}, \\
& -\frac{1}{2}-\frac{i}{2}-\frac{3 j}{2}-\frac{k}{2}, \frac{1}{2}-\frac{3 i}{2}+\frac{j}{2}-\frac{3 k}{2}, 1+j+k,-1+j, \frac{1}{2}-\frac{i}{2}-\frac{3 j}{2}-\frac{3 k}{2}, \\
& -1+i+j-k,-2+j, 1-i+j+k, 1+k,-1+j-k, 1-i+k\} \text {. }
\end{aligned}
$$

Theorem 4.5. (1) If $0 \neq \beta \in \mathcal{H}_{\alpha}, N(\beta)=5$ and $\beta \mid \alpha$, then the set of the subgroup $\langle\beta\rangle$ generated by $\beta$ is a perfect $1-$ dominating set in $G_{\alpha}$.

(2) If $0 \neq \beta \in \mathcal{H}_{\alpha}, N(\beta)=7$ and $\beta \mid \alpha$, then the set of the subgroup $\langle\beta\rangle$ generated by $\beta$ is a perfect 2 -dominating set in $G_{\alpha}$.

Proof. 1. Let $N(\beta)=5$ and $\beta \mid \alpha$ We prove that $d_{\alpha}(\sigma, \tau) \geq 3$ for all $\sigma, \tau \in\langle\beta\rangle, \sigma \neq$ $\tau$. Since $\sigma$ and $\tau$ are the elements of $\langle\beta\rangle$, there are $\delta_{1}, \delta_{2}$ in $\mathcal{H}_{\alpha}$ such that $\sigma=\beta \delta_{1}$ and $\tau=\beta \delta_{2}$. Thus, we have

$$
d_{\alpha}(\sigma, \tau)=d_{\alpha}(\sigma-\tau, 0)=d_{\alpha}\left(\beta \delta_{1}-\beta \delta_{2}, 0\right)=d_{\alpha}(\beta \gamma, 0),
$$


where $\gamma=\delta_{1}-\delta_{2}(\bmod \alpha)$. Let us assume that

$$
d_{\alpha}(\sigma, \tau)=d_{\alpha}(\beta \gamma, 0)<3 .
$$

In this situation, there is an element $q$ in $\mathcal{H}_{\alpha}$ such that $\beta \gamma$ is equal to $q$ modulo $\alpha$, that is, $q=\beta \gamma(\bmod \alpha)$. According to Def. 6 , we get

$$
d_{\alpha}(\sigma, \tau)=d_{\alpha}(\sigma-\tau, 0)=d_{\alpha}(\beta \gamma, 0)=N(q)<3 .
$$

Since $q=\beta \gamma(\bmod \alpha)$ and $\beta$ is a left divisor of $\alpha$, we get

$$
\beta \gamma=q+\alpha \gamma_{1}, \alpha=\beta \gamma_{2},
$$

respectively, for some $\gamma, \gamma_{2} \in \mathcal{H}$. Thus, we obtain

$$
\begin{gathered}
\beta \gamma=q+\alpha \gamma_{1}=q+\left(\beta \gamma_{2}\right) \gamma_{1}=q+\beta\left(\gamma_{2} \gamma_{1}\right) \\
\Rightarrow q=\beta\left(\gamma-\gamma_{2} \gamma_{1}\right)
\end{gathered}
$$

and

$$
N\left(\gamma-\gamma_{2} \gamma_{1}\right)=\frac{N(q)}{N(\beta)} .
$$

But, this is contradict to the definition of the norm since $N(\beta)=5, N(q)<3$, so, $N\left(\gamma-\gamma_{2} \gamma_{1}\right)=\frac{N(q)}{N(\beta)} \notin \mathbb{Z}$. Hence, the proof is completed.

Here, note that $\sigma \neq \tau$, so, $N(q) \neq 0$. A similar proof can be obtained for the case 2 .

Theorem 4.6. Let us assume that there is a Hurwitz integer $\beta$ and let $N(\beta)^{2}$ denotes the number of Hurwitz integers which the norm of these integers is less than or equal to $t$. In this situation, there exists a perfect $t$-dominating set in $\mathcal{H}_{\alpha}$ if there exists a prime $p$ such that the norm of $\beta$ is equal to $p$.

It is well known that there is a natural way of defining perfect error-correcting group codes with length one by means of perfect dominating sets over known graphs. Some examples associated with this topic can be seen in [13].

We use Mathematica software program to determine the codes given in this paper. As an illustration, we give the following algorithm in Fig. 5 for getting perfect 1 -dominating set. In that algorithm, we take $\alpha=1+3 i+2 j+k$ and $\beta=2+i$. The first column of the table "K", one can see the table "K" when the program runs, denotes the elements of $\mathcal{H}_{\alpha}$, the second column denotes the elements which dominated by the elements of $\langle\beta\rangle$, the set "SW1" denotes the set $\mathcal{E} \cup\{0\}$, the set "B1 $\gamma$ " denotes Hurwitz integers which the norm between $\gamma \in\langle\beta\rangle$ and these elements is 1. Note that any Hurwitz integer $a_{0}+a_{1} i+a_{2} j+a_{3} k$ is shown as Quaternion $\left[a_{0}, a_{1}, a_{2}, a_{3}\right]$ in Mathematica. We don't show the outputs since they takes up too much space in the paper.

Fig. 4 shows the graph $C_{225}(13,14, \ldots, 24)$ which it is isomorphic to the graph given in Fig. 3. The diameter of the graph $C_{225}(13,14, \ldots, 24)$ is 5 . Using the technic presented in this paper, one can construct a code that the minimum distance of the code is less than or equal to 5 . The graph immediately gives the minimum distance of a code presented in this paper.

Fig. 3 shows representation of the Hurwitz graph generated by $\alpha=1+3 i+2 j+k$. In the figure, points labeled red denotes the set $\langle\beta\rangle$. Note all vertexes are not given in the figure. The vertexes given in the figure shows one twelfth of all vertexes. 


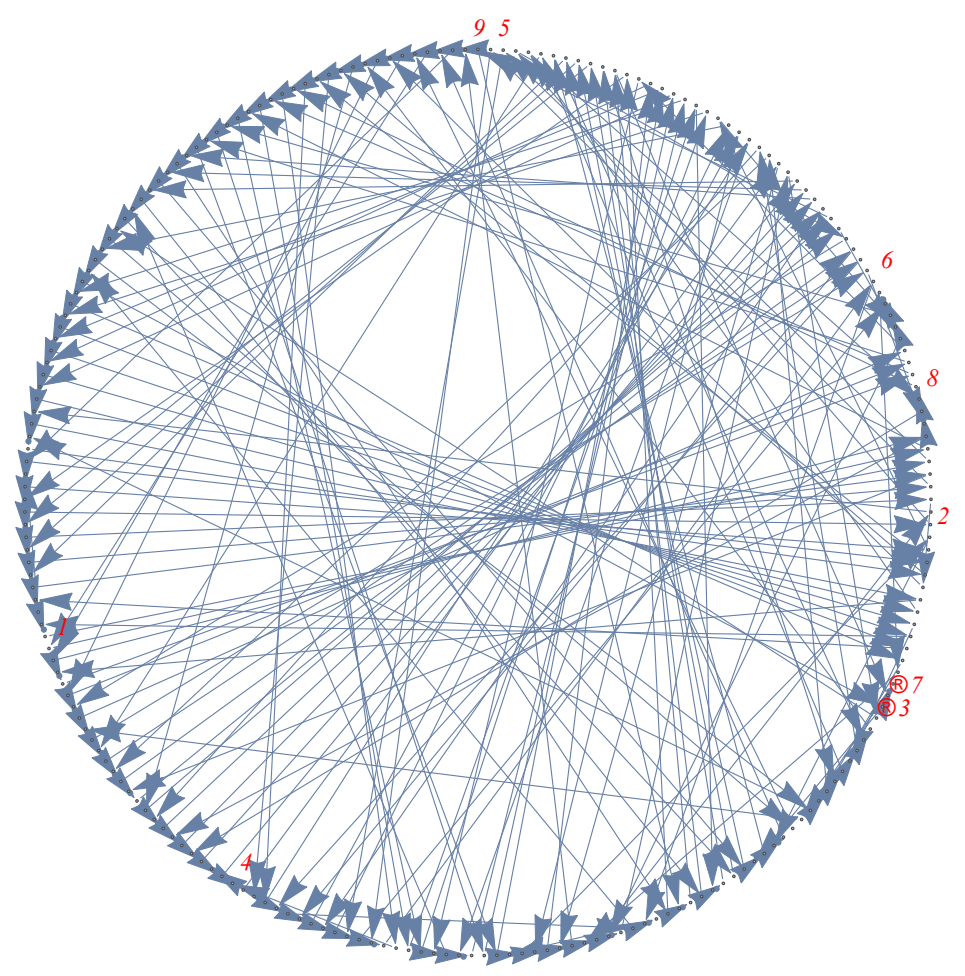

FiguRE 3. A representation of the Hurwitz graph generated by $\alpha=1+3 i+2 j+k$

Table I: Some perfect code parameters

\begin{tabular}{|c|c|c|}
\hline$\alpha$ & $\beta$ & $t$ \\
\hline $1+2 i-3 j+k$ & $i-2 k$ & 1 \\
\hline$-\frac{5}{2}+\frac{5 i}{2}+\frac{3 j}{2}+\frac{k}{2}$ & $\frac{3}{2}+\frac{i}{2}+\frac{3 j}{2}+\frac{k}{2}$ & 1 \\
\hline$-\frac{3}{2}+\frac{i}{2}-\frac{5 j}{2}+\frac{7 k}{2}$ & $\frac{1}{2}+\frac{i}{2}+\frac{j}{2}+\frac{5 k}{2}$ & 2 \\
\hline$\frac{3}{2}+\frac{5 i}{2}+\frac{9 j}{2}+\frac{5 k}{2}$ & $\frac{3}{2}+\frac{3 i}{2}+\frac{3 j}{2}+\frac{k}{2}$ & 2 \\
\hline $3+4 i+3 j+k$ & $2+i+j+k$ & 2 \\
\hline$-4+5 i+5 j+5 k$ & $1+2 i+2 j+2 k$ & 4 \\
\hline
\end{tabular}

Also, we give some perfect $t$-dominating sets, which directly are perfect codes at Table I.

\section{Some Comparisons}

In this section, we compare codes given in the present paper and some codes given in literature in terms of average energy and bandwidth occupancy. Firstly, we give a comparison associated with average energy. The average energy calculated as:

$$
E_{\text {avg }}=\frac{1}{M} \sum_{r=0}^{M-1}\left|q_{r}\right|^{2},
$$




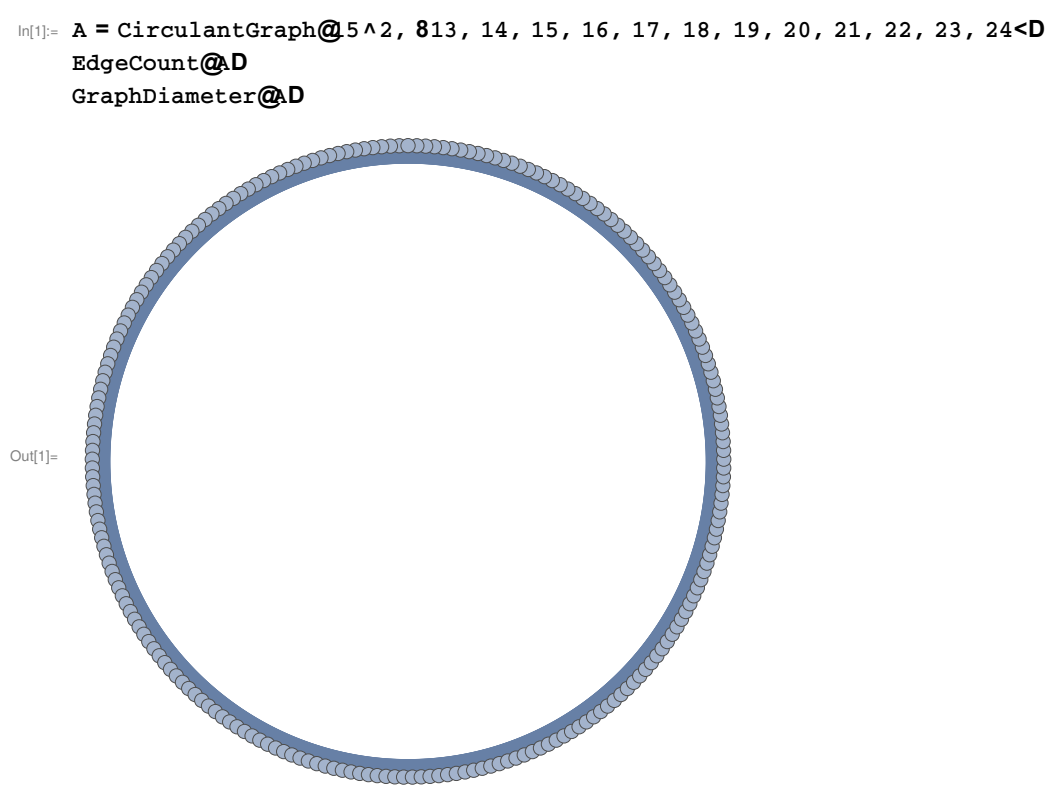

Out[2] $=2700$

Out $[3]=5$

Figure 4 . The graph $C_{225}(13,14, \ldots, 24)$

where $q_{r}$ is in signal space and it has a magnitude (distance from the origin) of $\left|q_{r}\right|=\sqrt{q_{r, 0}^{2}+q_{r, 1}^{2}+q_{r, 2}^{2}+q_{r, 3}^{2}}$ and $M$ denotes the number of the constellation.

\begin{tabular}{|c|c|c|c|c|}
\multicolumn{7}{|c|}{ Table II: Average Energy Comparison } \\
\hline$\alpha$ & $N(\alpha)$ & Base group & Number of constellation & $E_{\text {avg }}$ \\
\hline $2+i$ & 5 & $\mathbb{Z}[i]_{2+i}$ & 5 & 0.8 \\
\hline $2+i$ & 5 & $\mathcal{H}_{2+i}$ & 25 & 0.96 \\
\hline $3+4 i$ & 25 & $\mathbb{Z}[i]_{3+4 i}$ & 25 & 4.16 \\
\hline 15 & $1+3 i+2 j+k$ & $\mathcal{H}_{1+3 i+2 j+k}$ & 225 & 3.27 \\
\hline $3+4 i$ & 25 & $\mathcal{H}_{3+4 i}$ & 625 & 5.30 \\
\hline $3+4 i$ & 25 & $\mathbb{Z}[i]_{3+4 i}^{2}$ & 625 & 8.32 \\
\hline
\end{tabular}

Table II shows that the average energy of codes over Hurwitz integers is better than the average energy of codes over Gaussian integers.

Secondly, we compare codes in terms of bandwidth occupancy. One of the most important parameter of analog/digital communication systems is bandwidth. So far, various modulation and coding techniques are developed to provide bandwidth efficiency. As we know from the communication theory, to attain the equal channel capacity required bandwidth must be higher when the codeword number increases $[16,17]$. Bandwidth occupancy $B W$ is calculates as

$$
B W=\frac{C_{a}}{\log (1+S N R)},
$$

where $C_{a}$ and $S N R$ denote the channel capacity and signal-to-noise ratio, respectively. The $B W$ of codes over Hurwitz integers is better than the $B W$ of codes over 


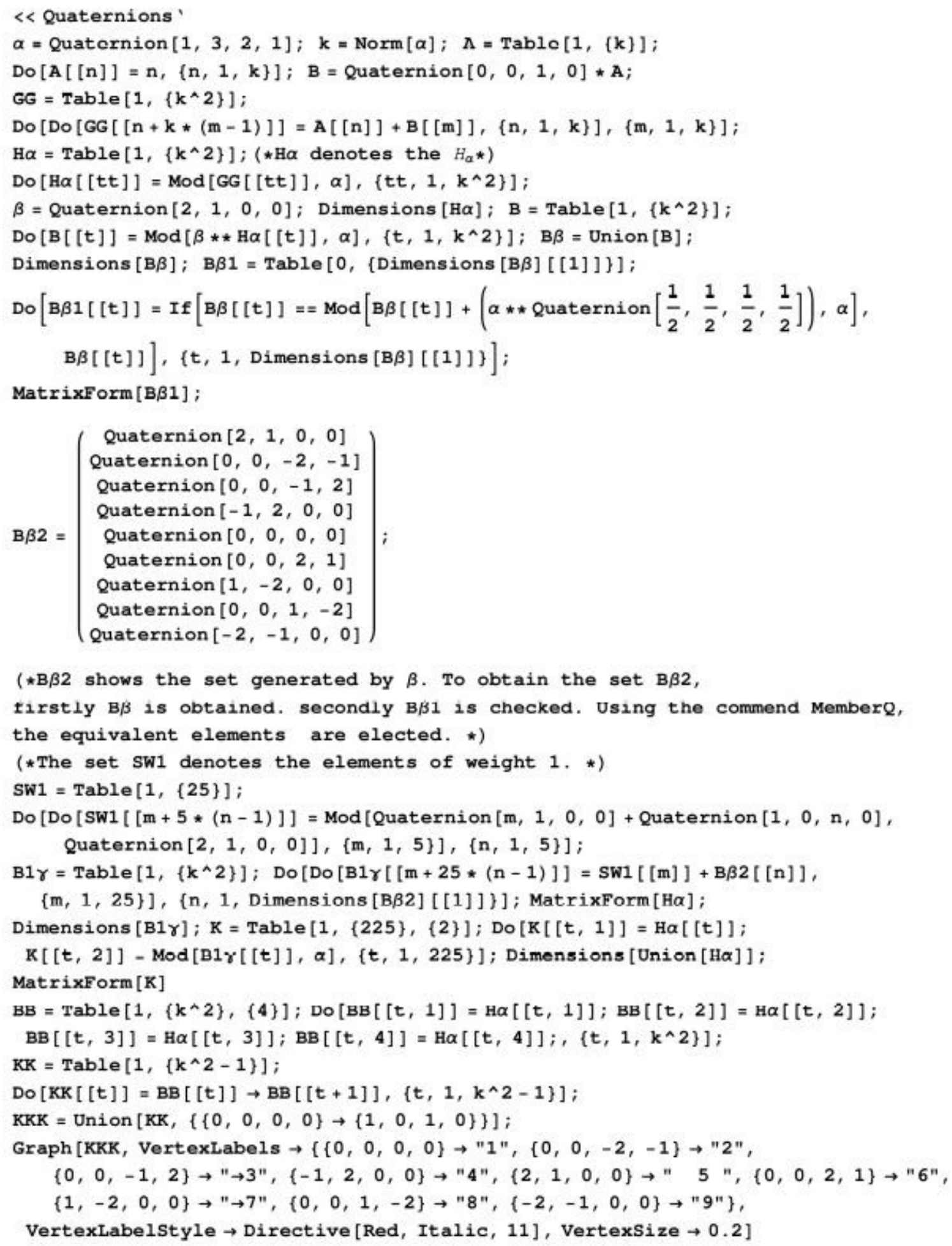


Gaussian integers since the number of codewords in a code over Hurwitz integers is equal to square of the number of the codewords in a code over Gaussian integers for the same integer $N(\alpha)$ and the same length $n$. For example, a code $C$ has 625 codewords in Hurwitz integers while a code $C$ has 25 codewords in Gaussian integers for the same integer $N(\alpha)=25, \alpha=3+4 i$, and the same length $n=1$.

\section{Conclusion}

The paper devotes a new family of $t$-error correcting perfect codes over Hurwitz integers. Using perfect $t$-dominating sets over the circulant graphs, these perfect codes are constructed. Codes given in the present paper and some codes given in literature in terms of average energy and bandwidth occupancy are compared. It is shows that the average energy of codes over Hurwitz integers is better than the average energy of codes over Gaussian integers.

\section{Acknowledgments}

The authors would like to thank the reviewers and editors of Journal of Universal Mathematics.

\section{Funding}

The work was supported by TÜBITAK (The Scientific and Technical Research Council of TURKEY) with project number 116F318.

\section{The Declaration of Conflict of Interest/ Common Interest}

The author(s) declared that no conflict of interest or common interest

\section{The Declaration of Ethics Committee Approval}

This study does not be necessary ethical committee permission or any special permission.

\section{The Declaration of Research and Publication Ethics}

The author(s) declared that they comply with the scientific, ethical, and citation rules of Journal of Universal Mathematics in all processes of the study and that they do not make any falsification on the data collected. Besides, the author(s) declared that Journal of Universal Mathematics and its editorial board have no responsibility for any ethical violations that may be encountered and this study has not been evaluated in any academic publication environment other than Journal of Universal Mathematics.

\section{REFERENCES}

[1] M. Güzeltepe, Codes over Hurwitz integers, Discrete Math. 313, pp. 704-714, (2013).

[2] M. Güzeltepe, A. Altınel, Perfect 1-error-correcting Hurwitz weight codes, Math. Commun. 22, pp. 265-272, (2017).

[3] M. Güzeltepe, O. Heden, Perfect Mannheim, Lipschitz and Hurwitz weight codes, Math. Commun. 19, pp. 253-276, (2014).

[4] R.W. Hamming, Error detecting and error correcting codes, Bell System Technical Journal 29, pp. 147-160, (1950).

[5] O. Heden, A new construction of group and nongroup perfect codes, Information and Control 34, pp. 314-323, (1977). 
[6] O. Heden, M. Güzeltepe, On perfect 1- $\varepsilon$-error-correcting codes, Math. Commun. 20, pp. 23-35, (2015).

[7] O. Heden, M. Güzeltepe, Perfect 1-error-correcting Lipschitz weight codes, Math. Commun. 21, pp. 23-30, (2016).

[8] K. Huber, Codes over Gaussian integers, IEEE Trans. Inform. Theory 40, pp. 207-216, (1994).

[9] C.Y. Lee, Some properties of non-binary error correcting codes, IEEE Trans. Inform. Theory 4(), pp. 77-82, (1958).

[10] B. B. Lindström, On group and nongroup perfect codes in $q$ symbols, Math. Scand. 25, pp. 149-158, (1969).

[11] C. Martínez, E. Stafford, R. Beivide, E. Gabidulin, Perfect codes over Lipschitz integers, in: Proc. IEEE Int. Symp. Information Theory, Nice, pp. 1366-1370, (2007).

[12] C. Martínez, R. Beivide, E. Gabidulin, Perfect codes from Cayley graphs over Lipschitz integers, IEEE Trans. Inf. Theory 55, pp. 3552-3562, (2009).

[13] C. Martínez, R. Beivide, E. Gabidulin, Perfect codes for metrics induced by circulant graphs, IEEE Trans. Inf. Theory 53, pp. 3042-3052, (2007).

[14] J. Schönheim, On linear and nonlinear, single-error-correcting $q$-nary perfect codes, Information and Control 12, pp. 23-26, (1968).

[15] Y.L. Vasil'ev, On nongroup close-packed codes, Problemi Tekhn. Kibernet. Robot. 8, pp. 337-339, (1962).

[16] J. G. Proakis, M. Salehi, Communications Systems Engineering, Second Edition, Prentice Hall, (2001).

[17] S. Lin, D. J. Costello, Jr., Error Control Coding, Second Edition, Prentice Hall, (2001).

(Author 1) Sakarya University, Department of Mathematics, 54187, Sakarya/TURKEY Email address: mguzeltepe@sakarya.edu.tr

(Author 2) Sakarya University, Department of Mathematics, 54187, Sakarya/TURKEy

Email address: gokhanguner54@hotmail.com 